\title{
Care pathways and treatment patterns for patients with heart failure in China: results from a cross-sectional survey
}

This article was published in the following Dove Press journal:

Drug Design, Development and Therapy

\author{
James DS Jackson' \\ Sarah E Cotton' \\ Sara Bruce Wirta ${ }^{2}$ \\ Catia C Proenca ${ }^{3}$ \\ Milun Zhang ${ }^{4}$ \\ Raquel Lahoz ${ }^{5}$ \\ Bogdan Balas ${ }^{5}$ \\ Frederico J Calado ${ }^{5}$
}

'Real World Research, Adelphi Real World, Bollington, UK; ${ }^{2}$ Real World Evidence, Cardio-Metabolic Franchise, Novartis Sweden AB, Stockholm, Sweden; ${ }^{3}$ Wellmera AG, Basel, Switzerland; ${ }^{4}$ Health Economics and Outcomes Research, Novartis Pharma China, Beijing, China; ${ }^{5}$ Medical Affairs, Cardio-Metabolic Franchise, Novartis Pharma AG, Basel, Switzerland
Correspondence: James DS Jackson Adelphi Real World, Adelphi Mill, Grimshaw Lane, Bollington, Cheshire SKIO 5JB, UK

Tel +44 I62 $557737 \mid$

Fax +44I62 5577294

Email james.jackson@adelphigroup.com
Purpose: The objective of this study was to describe the clinical care pathways, management and treatment patterns, and hospitalizations for patients with heart failure (HF) in China.

Subjects and methods: A cross-sectional survey of cardiologists and their patients with HF was conducted. Patient record forms were completed by 150 cardiologists for 10 consecutive patients. Patients for whom a patient record form was completed were invited to complete a patient self-completion questionnaire.

Results: Most of the 1,500 patients (mean [SD] age 66 [10] years; 55\% male) included in the study received care in tier-2 and -3 hospitals in large cities. Cardiologists were responsible for initial consultation, diagnosis, and treatment of patients with HF. The use of guidelinerecommended diagnostics was high. However, guideline-recommended double- and triplecombination therapy was received by only $51 \%$ and $18 \%$ of patients, respectively. In total, $20 \%$ of patients with HF reported that they were not consulted on the choice of therapy. Concordance was high ( $\geq 80 \%$ ) between matched cardiologist and patient pairs for the occurrence of side effects, while cardiologists more often under- than overreported the occurrence of side effects of treatment reported by patients.

Conclusion: The management of HF was predominantly overseen by cardiologists. The use of diagnostic tests was high, but the use of guideline-recommended treatment was low in this population. Improved communication between patients and cardiologists is essential to optimize treatment decision making and to increase awareness of treatment side effects.

Keywords: heart failure, disease management, patient preference, treatment satisfaction, real-world evidence

\section{Introduction}

Heart failure (HF) represents a major clinical and public health problem worldwide, affecting 62 million people. ${ }^{1}$ In China, there are $\sim 4.5$ million individuals with HF, equating to a prevalence of $0.9 \%{ }^{2} \mathrm{HF}$ can be classified as HF with reduced ejection fraction (HFrEF) or HF with preserved ejection fraction (HFpEF). HFrEF and HFpEF each comprise $50 \%$ of all cases of $\mathrm{HF}$ in China. ${ }^{3-5}$

China has one of the world's largest health care systems, with $\sim 28,000$ hospitals, a third of which are tier-2 hospitals (medium-sized regional hospitals serving mediumsized cities) or tier-3 hospitals (large municipal hospitals providing care at a national level). ${ }^{6}$ In Western countries, care for patients with HF is generally provided by hospital- or office-based cardiologists and/or family physicians. ${ }^{7}$ Patients usually have a family physician who refers them to a more specialized physician when further input is required. ${ }^{8}$ Information on the typical care pathway of patients with HF in 
China has not been published in the literature. Treatment is based on recommendations made by the Chinese Society of Cardiology of the Chinese Medical Association, ${ }^{9}$ which recommend that routine examination for the diagnosis of patients with HF should comprise an echocardiogram, an electrocardiogram, laboratory tests (complete blood count, urinalysis, blood chemistry, fasting glucose level, glycated hemoglobin level, and thyroid function), levels of biomarkers (B-type natriuretic peptide or N-terminal pro-B-type natriuretic peptide), and an X-ray.

Most clinical trials published after 1990 report efficacy data for patients with HFrEF, based predominantly on a left ventricular ejection fraction threshold of $40 \%$ or less. For this reason, the Chinese Society of Cardiology guidelines mainly provide recommendations for the pharmacological treatment of this patient population (although left ventricular ejection fraction thresholds of $<50 \%$ and $\geq 50 \%$ are suggested for HFrEF and HFpEF, respectively, and these definitions are not unified). ${ }^{9}$ The guidelines recommend angiotensin-converting enzyme inhibitors (ACEis), angiotensin receptor blockers (ARBs), $\beta$-blockers (BBs), mineralocorticoid receptor antagonists (MRAs), digoxin, diuretics, and nitrates for patients with HFrEF.

These drugs are available in most countries, including China; however, treatment gaps in the management of patients with HF still exist. ${ }^{10}$ Several large, real-world studies of treatment patterns for HF in China conducted in the early $2000 \mathrm{~s}^{3-5,11,12}$ showed that nitrates, digitalis, and diuretics were the main drugs used to treat HF; the proportions of patients using these agents were $30 \%-91 \%, 40 \%-72 \%$, and 49\%-77\%, respectively. ACEis and BBs were less widely used, although their use increased between the 1980 s and the 2000 s (from $9 \%$ to $19 \%$ of patients for BBs and from $14 \%$ to $40 \%$ for ACEis). ${ }^{11,13}$ A more recent survey conducted in 2009 of physicians from primary care hospitals across 17 areas of China observed trends similar to those of the earlier studies in terms of high use of diuretics and digitalis, although BBs and ACEis were more widely used in this study ( $40 \%$ and $80 \%$ of patients, respectively), likely reflecting increased use of these drug classes over time. ${ }^{14}$

The aim of the current cross-sectional study of patients with HF treated by cardiologists in a real-world setting in China was threefold: 1) to describe the patient pathway in terms of how patients with HF interact with and are treated by health care professionals; 2 ) to evaluate the use of treatment classes and how treatment decisions are being made; and 3 ) to understand the burden of patients with HF in terms of adverse events and hospitalizations.

\section{Subjects and methods Study design}

Data were drawn from the Adelphi HF Disease Specific Programme (DSP), a cross-sectional survey of cardiologists, their patients with HF, and those patients' informal caregivers, conducted in a real-world setting in China in 2016. Full details of the methodology are provided elsewhere. ${ }^{15,16}$ Briefly, a DSP comprises three key phases: preparatory, data collection, and data processing/analysis. No formal validation procedure was undertaken; however, all numerical data were double entered.

\section{Preparatory phase}

\section{Development of fieldwork materials}

Four questionnaires were developed to inform the DSP - a physician survey, a patient record form (PRF), a patient self-completion questionnaire (PSC), and a caregiver selfcompletion questionnaire. The physician survey was used in face-to-face interviews with cardiologists. PRFs were completed by the cardiologists for their patients presenting with HF using data from medical records. PSCs were completed by the same patients, and caregiver self-completion questionnaires were completed by the informal caregivers of the patients (results gathered from the caregiver selfcompletion questionnaires have been reported by Jackson et al). ${ }^{16}$ The questionnaires were developed empirically with input from experts in HF care; the concepts deemed most relevant to patients were selected empirically, and their pharmacometric properties were not systematically assessed. The questionnaires were developed in English and then translated into Chinese by a local DSP fieldwork agency. A second independent UK-based translation agency verified the translated materials. Samples of the questionnaires are available on request.

\section{Participant recruitment}

Cardiologists were identified from the public lists of health care professionals and were invited to participate in the DSP based on predefined eligibility criteria defined elsewhere. ${ }^{16}$ The first 150 cardiologists who met these criteria and agreed to participate in the study were enrolled and asked to complete PRFs for the next 10 patients they saw who presented with HF, immediately after each consultation. The same patients were invited to complete PSCs at the practice, independently of their cardiologist and immediately after their consultation. Included patients gave informed consent to participate by ticking a box on the front page of the questionnaire to indicate 
that they have read the information provided and that they agree to take part in the study.

\section{Data collection phase}

For this analysis, the physician survey with the cardiologists was used to capture information on practice type and location. Information collected from the PRFs covered patient demographics; clinical characteristics; consultation history; scans/ investigations, physical examinations, and blood/urine tests during diagnosis and monitoring of HF; HF treatment; and all-cause and HF-related inpatient visits, outpatient visits, and emergency room visits (PRF questions pertaining to results presented herein are included in Supplementary materials. Appendix 1). Information gathered from the PSCs covered the duration of time between symptom onset and first visit to a physician (PSC questions pertaining to results presented herein are included in Supplementary materials, Appendix 1).

All responses were anonymized to preserve participant confidentiality and to avoid potential bias. The questionnaire applied in this study followed the European Pharmaceutical Market Research Association guidelines. ${ }^{17}$ The Code of Conduct states that within this context, ethical approval is not necessary, considering that the goal of research rather than being to test a hypothesis is to improve understanding rather than testing the hypotheses. The research was conducted in accordance with the US Health Insurance Portability and Accountability Act 1996 and European equivalents. ${ }^{17,18}$

\section{Data processing/analysis phase}

For the purposes of analysis and interpretation, HFrEF was defined as left ventricular ejection fraction $<50 \%$ and $\mathrm{HFpEF}$ as $\geq 50 \%$, in accordance with the Chinese Society of Cardiology guidelines. ${ }^{9}$ Descriptive statistics were used for all baseline characteristics and outcomes of interest with the analyses conducted using the software package QPSMR Reflect, version 2007.1g (QPSMR Ltd, Wallingford, UK). Continuous variables were presented as mean $\pm \mathrm{SD}$, or median (in the case of health care visit frequency), while categorical variables were presented as number (n) and percentage (\%) of patients. For the patient-physician paired analyses, concordance was calculated as: $\mathrm{n}$ (concordant absence of symptom/adverse event + concordant presence of symptom/adverse event)/ $\mathrm{N}$ (total numbers of matched pairs). Furthermore, the patient perspective was taken to evaluate physician underreporting (side effect reported by patient but not physician/total number of patients reporting side effect) and physician overreporting (side effect reported by physician but not patient/total number of patients not reporting side effect).

\section{Results}

\section{Study population}

The study comprised 150 cardiologists and 1,500 patients. Almost two-thirds $(62 \%, n=933)$ of the patients for whom a PRF was completed also completed a PSC. ${ }^{16}$

\section{Cardiologist demographics}

Of the 150 cardiologists, the majority (60\%) were based in Beijing, Shanghai, or Guangdong (Figure S1). Most (91\%) of the cardiologists worked only in a hospital; of these, $69 \%$ worked in tier-3 hospitals.

\section{Patient demographics and baseline clinical characteristics}

Overall, $55 \%$ of the patients were male, and their mean (SD) age was 66 (10) years in both the PRF and PSC subsamples. The baseline clinical characteristics for patients are shown in Table 1, separately for the PRF and PCS patient samples.

\section{Patient pathway}

\section{Reasons for consultation and physician specialties}

Based on data from the PRFs, patients mainly consulted physicians for a routine follow-up or a repeat prescription (46\%),

Table I Baseline clinical characteristics

\begin{tabular}{|c|c|c|}
\hline \multirow[t]{2}{*}{ Clinical characteristic } & \multirow{2}{*}{$\begin{array}{l}\text { PRF } \\
n=I, 500\end{array}$} & \multirow{2}{*}{$\begin{array}{l}\text { PSC } \\
n=933\end{array}$} \\
\hline & & \\
\hline \multicolumn{3}{|c|}{ New York Heart Association functional class $(n=1,494)$} \\
\hline I & $377(25)$ & $217(23)$ \\
\hline II & $698(47)$ & $449(48)$ \\
\hline III & $355(24)$ & $230(25)$ \\
\hline IV & $64(4)$ & $34(4)$ \\
\hline Current left ventricular ejection fraction & $\mathrm{n}=\mathrm{I}, 298$ & $n=813$ \\
\hline Mean (SD) & $50(\mathrm{II})$ & $50(\mathrm{II})$ \\
\hline Comorbidity & $\mathrm{n}=\mathrm{I}, 500$ & $n=933$ \\
\hline Hypertension & I, I87 (79) & 74I (79) \\
\hline Angina & $676(45)$ & $427(46)$ \\
\hline Sleeping problems & $569(38)$ & $361(39)$ \\
\hline Hyperlipidemia & $502(33)$ & $306(33)$ \\
\hline Anxiety & $415(28)$ & $273(29)$ \\
\hline Underlying cause of HF & $\mathrm{n}=1,500$ & $n=933$ \\
\hline Hypertension & $986(66)$ & $621(67)$ \\
\hline Coronary heart disease/myocardial infarction & $650(43)$ & $410(44)$ \\
\hline Arrhythmia & $37 \mid(25)$ & $231(25)$ \\
\hline Diabetes & $266(18)$ & $159(17)$ \\
\hline Atrial fibrillation & $227(15)$ & $130(14)$ \\
\hline
\end{tabular}

Notes: Values presented are $\mathrm{n}(\%)$, unless otherwise stated; percentages are calculated including missing data and are subject to rounding. Data were obtained from patient record forms.

Abbreviations: HF, heart failure; PRF, patient record form; PSC, patient selfcompletion questionnaire. 
or for a diagnosis of HF or testing of HF symptoms (44\%). Few patients (1\%) scheduled a consultation to discuss HF treatment (ie, because their treatment was not working or because of the occurrence of side effects), as shown in Figure S2.

\section{Use of scans/investigations, physical examinations, and blood/urine tests during diagnosis and monitoring of HF}

The use of scans/investigations, physical examinations, and blood/urine tests during diagnosis and monitoring of patients with HF $(n=1,219-1,474)$ is shown in Figure 1. In general, scans/investigations were more commonly used during diagnosis of HF than when monitoring it, with the exception of electrocardiograms, which were more commonly used when monitoring HF than for diagnosing it (78\% vs $63 \%$ of patients, respectively). Almost all patients (92\%) had undergone an echocardiogram during their diagnosis of HF. Similarly, examination of physical signs was generally more commonly used during diagnosis than monitoring; assessment of heart rate was the most common physical sign examined during HF diagnosis (82\%), while assessment of blood pressure was the most common physical sign used when monitoring HF (94\%). B-type natriuretic peptide and N-terminal pro-B-type natriuretic peptide were the most common blood/urine markers used to diagnose HF ( $69 \%$ and $66 \%$, respectively). Total cholesterol $(68 \%)$ and triglyceride $(62 \%)$ tests were most frequently performed during patient monitoring, followed by B-type natriuretic peptide (56\%), fasting glucose $(55 \%)$, glycated hemoglobin (47\%), and serum creatinine (45\%).

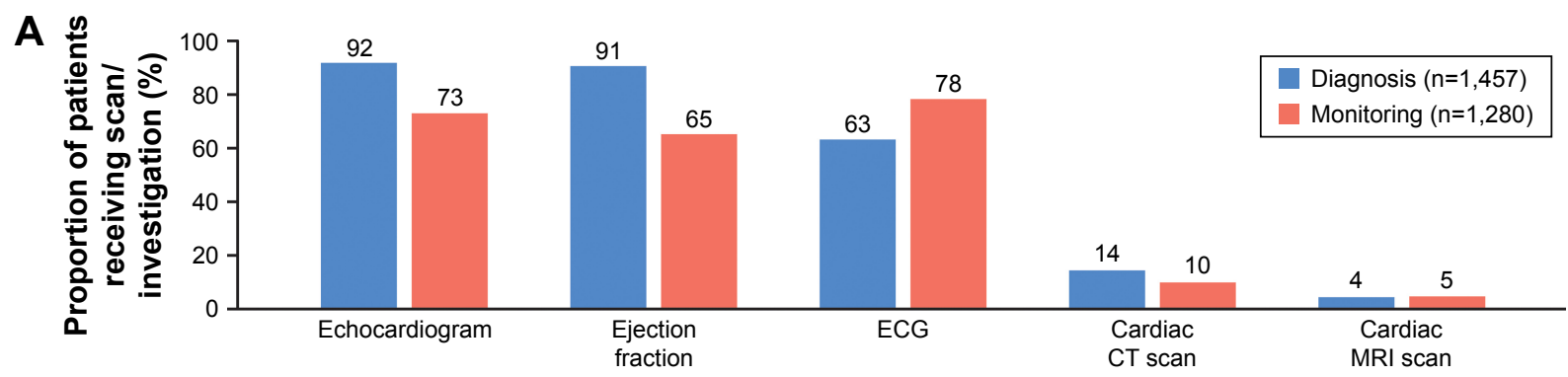

B
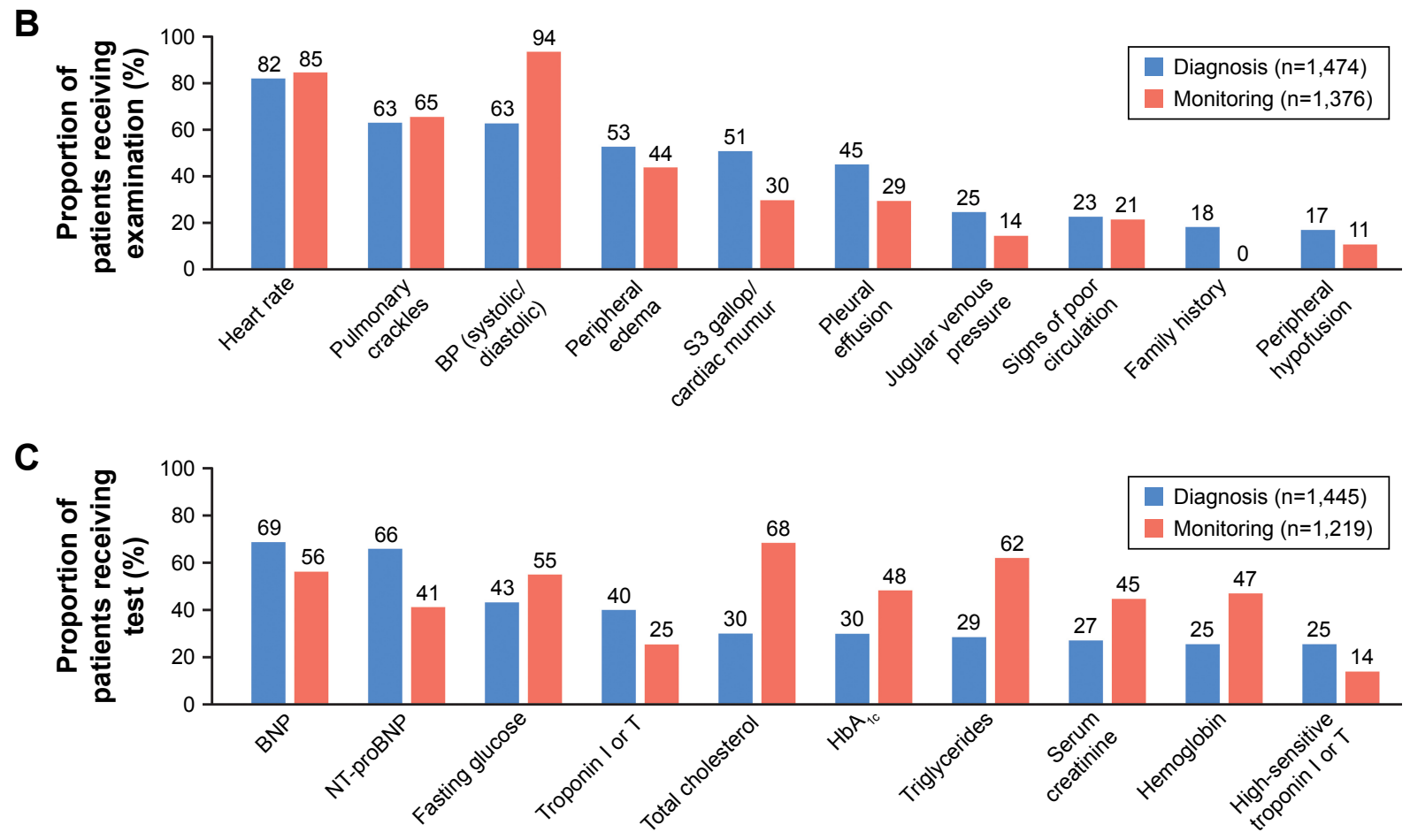

Figure I Use of (A) scans/investigations, (B) physical examinations, and (C) blood/urine tests during diagnosis and monitoring of patients' HF. Note: Data were obtained from patient record forms.

Abbreviations: BNP, B-type natriuretic peptide; BP, blood pressure; CT, computed tomography; ECG, electrocardiogram; HbA, glycated hemoglobin; HF, heart failure; MRI, magnetic resonance imaging; NT-proBNP, N-terminal pro-B-type natriuretic peptide. 


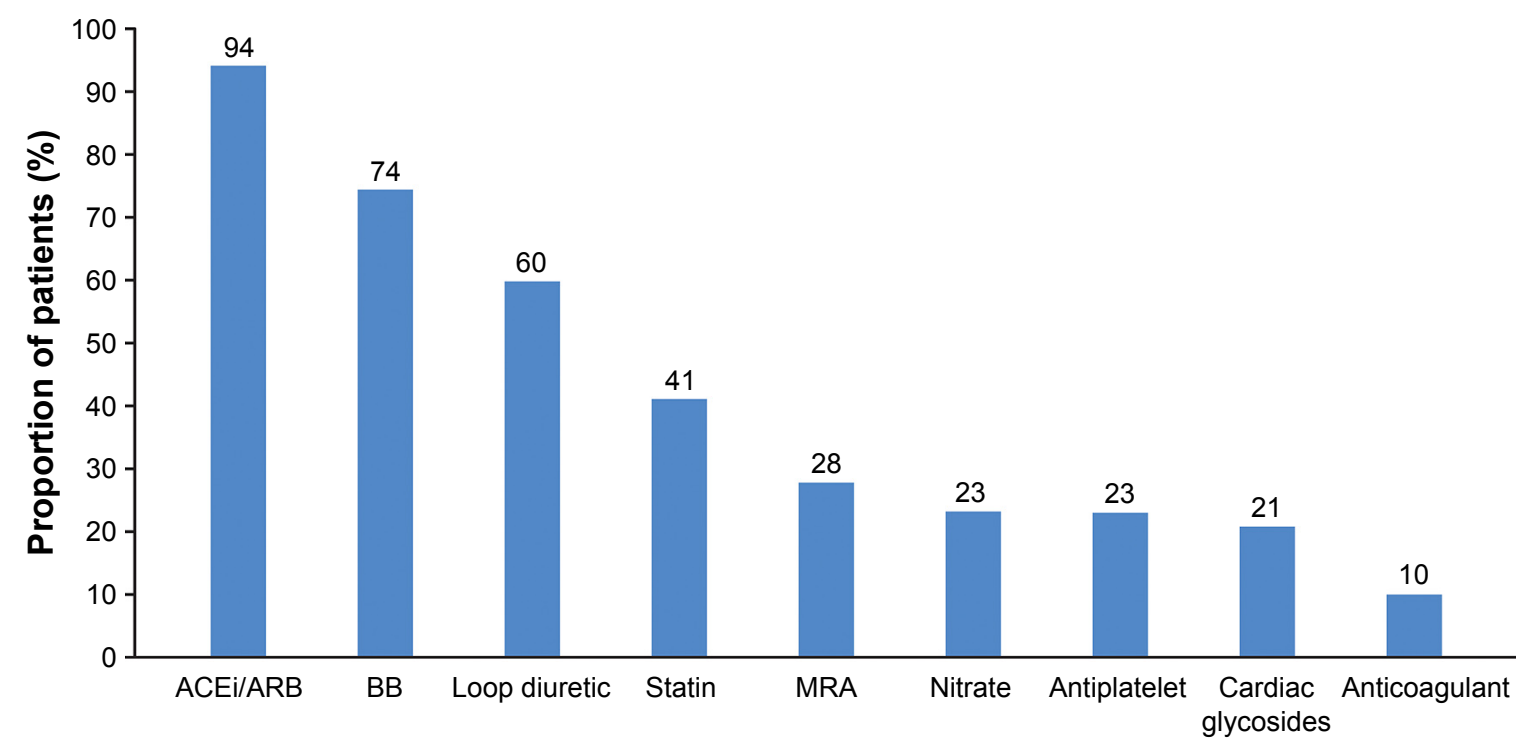

Figure 2 Most common HF treatment classes received by patients $(\mathrm{N}=\mathrm{I}, 498)$.

Note: Data were obtained from patient record forms.

Abbreviations: ACEi, angiotensin-converting enzyme inhibitor; ARB, angiotensin receptor blocker; BB, $\beta$-blocker; HF, heart failure; MRA, mineralocorticoid receptor antagonist.

\section{HF treatment patterns}

Information on HF treatment patterns was available for 1,498 patients. The most common treatment class used at the time the survey was conducted was ACEi/ARB (94\%), followed by BB (74\%) and loop diuretic $(60 \%)$, as shown in Figure 2. MRAs were received by $28 \%$ of patients. With regard to combination therapy, the largest proportion of patients $(51 \%)$ received $\mathrm{ACEi} / \mathrm{ARB}+\mathrm{BB}$, followed by $18 \%$ of patients who received ACEi/ARB+BB+MRA. More patients with HFrEF (21\%) than HFpEF (18\%), as well as those with more severe symptoms as determined by the New York Heart Association class (class I 10\%; class II-IV 24\%) received this combination, as shown in Table 2. The most common monotherapy received was ACEi or ARB (17\%), with higher proportions of patients with $\operatorname{HFrEF}(22 \%)$ than HFpEF (10\%) receiving these classes. BB monotherapy was uncommon (4\%).

Of 1,489 patients, cardiologists reported that the ability of the drug to lower blood pressure (reported for $75 \%$ of patients) was the most common reason for treatment choice, which might be explained by the fact that $79 \%$ of patients had hypertension. Cardiologists' own familiarity/experience with the drug was the second most common reason for treatment choice (reported for $73 \%$ of patients; Figure 3 ). Whether the choice of the drug was in accordance with the guidelines influenced cardiologists' choice for $57 \%$ of patients.

Table 2 Treatment class combinations received by patients with HF, stratified by HF phenotype and New York Heart Association functional class

\begin{tabular}{|c|c|c|c|c|c|}
\hline Treatment class combination & $\begin{array}{l}\text { Overall } \\
(\mathrm{N}=I, 498)\end{array}$ & $\begin{array}{l}\text { HFrEF } \\
(<\mathbf{5 0 \% )} \\
(n=677)\end{array}$ & $\begin{array}{l}\text { HFpEF } \\
(\geq 50 \%) \\
(n=621)\end{array}$ & $\begin{array}{l}\text { HFrEF New York } \\
\text { Heart Association } \\
\text { class I ( } n=\mid 45)\end{array}$ & $\begin{array}{l}\text { HFrEF New York } \\
\text { Heart Association } \\
\text { class II-IV }(n=530)\end{array}$ \\
\hline ACEi/ARB monotherapy $\left( \pm\right.$ others $\left.^{\mathrm{a}}\right)$ & $259(17)$ & $150(22)$ & $60(10)$ & $46(32)$ & $103(19)$ \\
\hline BB monotherapy ( \pm others ${ }^{\mathrm{a}}$ ) & $57(4)$ & $18(3)$ & $33(5)$ & $4(3)$ & $14(3)$ \\
\hline MRA monotherapy ( \pm others $\left.{ }^{a}\right)$ & $6(0)$ & $3(0)$ & $3(0)$ & $0(0)$ & $3(2)$ \\
\hline $\mathrm{ACEi}+\mathrm{ARB} \pm$ others $^{\mathrm{a}}$ & $132(9)$ & $89(13)$ & $20(3)$ & $38(26)$ & $51(10)$ \\
\hline $\mathrm{ACEi} / \mathrm{ARB}+\mathrm{BB}\left(\right.$ no MRA) \pm others $^{\mathrm{a}}$ & $76 I(5 I)$ & $304(45)$ & $370(60)$ & $74(5 \mathrm{I})$ & $229(43)$ \\
\hline ACEi/ARB+MRA (no BB) \pm others $^{a}$ & $113(8)$ & $50(7)$ & $28(5)$ & $6(4)$ & $44(8)$ \\
\hline $\mathrm{ACEi} / \mathrm{ARB}+\mathrm{BB}+\mathrm{MRA} \pm$ others & $277(18)$ & $143(2 \mid)$ & $113(18)$ & $14(10)$ & $129(28)$ \\
\hline
\end{tabular}

Notes: Values presented are $\mathrm{n}(\%)$, unless otherwise stated; percentages are calculated following the exclusion of missing data and are subject to rounding. Treatment groups are not mutually exclusive. Data were obtained from patient record forms. alncludes therapy unrelated to treating $\mathrm{HF}$ and excludes other $\mathrm{HF}$ treatments.

Abbreviations: ACEi, angiotensin-converting enzyme inhibitor; ARB, angiotensin receptor blocker; BB, $\beta$-blocker; HF, heart failure; HFpEF, heart failure with preserved ejection fraction; HFrEF, heart failure with reduced ejection fraction; MRA, mineralocorticoid receptor antagonist. 


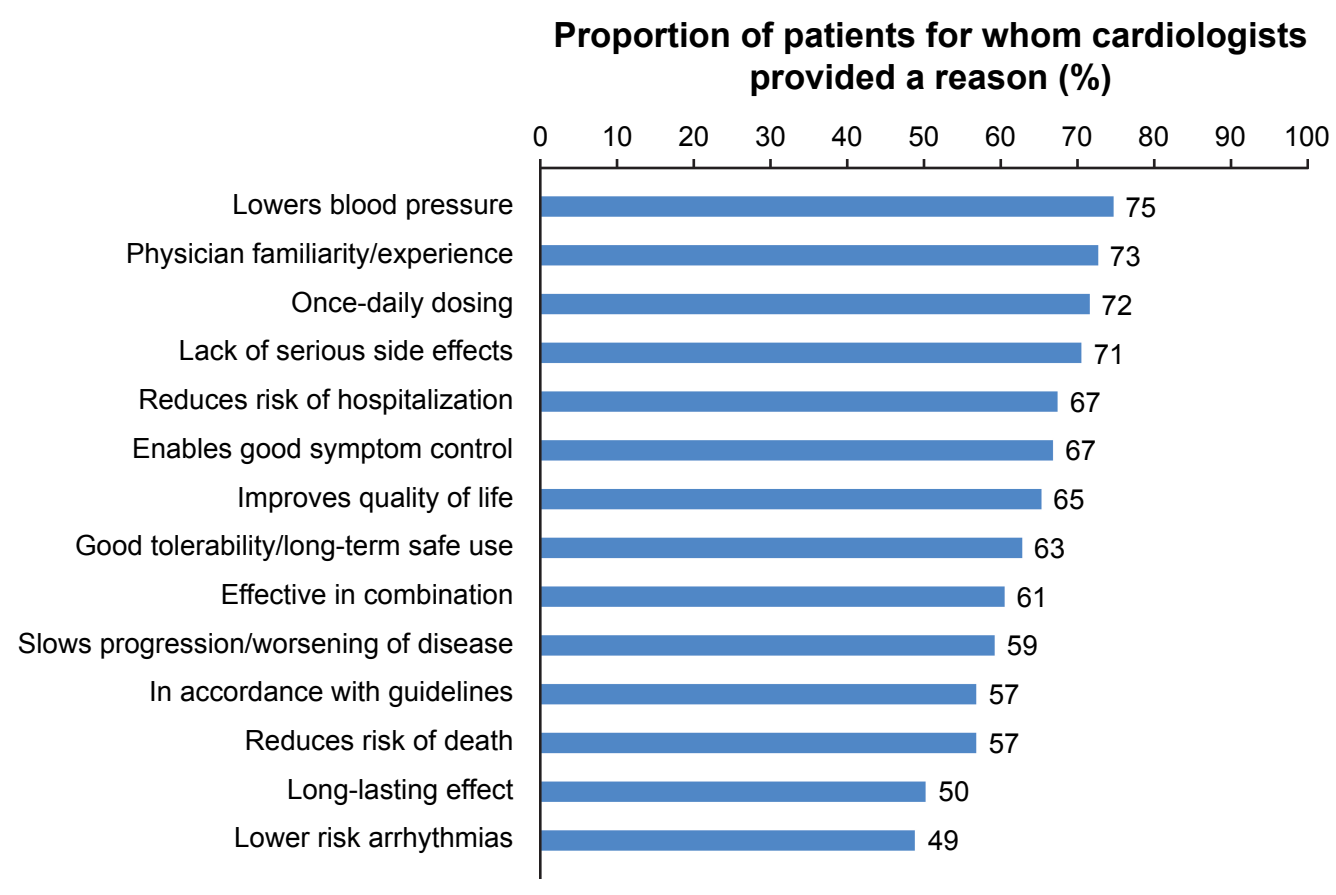

Figure 3 Cardiologists' most common reasons for HF treatment choice $(\mathrm{N}=\mathrm{I}, 489)$. Note: Data were obtained from patient record forms.

Abbreviation: HF, heart failure.

\section{Treatment decision making}

A high level of concordance (84\%) between matched cardiologist and patient pairs $(n=842)$ was observed for the degree of patient input into treatment decisions. In total, $20 \%$ of patients with HF reported that they were not consulted on the choice of therapy, and $71 \%$ of matched cardiologist and patient pairs reported that treatment choice was based on a discussion, while the cardiologist had the final call (Table 3).

\section{Treatment satisfaction}

Concordance was high between matched cardiologist and patient pairs $(\mathrm{n}=842)$ for the occurrence of side effects, ranging from $80 \%$ (fatigue/tiredness and dizziness) to $100 \%$ (numbness/tingling on skin and sexual dysfunction), as shown in Figure 4A. Individual side effects were reported by $0 \%-22 \%$ of patients (Figure $4 \mathrm{~B}$ ). Cardiologists more often under- than overreported the occurrence of side effects of treatment reported by patients (Figure 4B).

\section{Hospitalizations}

In China, doctors do not have access to patients' medical records outside of the hospital in which they see the patient. Therefore, the number of hospitalizations recorded by cardiologists in this study covered only hospitalizations that occurred at their particular hospital. Information on allcause and HF-related inpatient, outpatient, and emergency room visits was available for $1,278-1,490$ patients. In the 12 months before the survey, $55 \%$ and $49 \%$ of patients had $\geq 1$ inpatient visit to the hospital for any reason (median=1 visit)

Table 3 Concordance of patients influence over treatment decision (matched patient vs physician responses)

\begin{tabular}{|c|c|c|c|c|}
\hline \multirow{2}{*}{$\begin{array}{l}\text { Patient responses PSC, } \\
\text { n (\%) }\end{array}$} & \multicolumn{4}{|c|}{ Physician response PRF, n (\%) } \\
\hline & $\begin{array}{l}\text { Dr decision, } \\
\text { no discussion }\end{array}$ & $\begin{array}{l}\text { Discussion, } \\
\text { Dr has } \\
\text { final call }\end{array}$ & $\begin{array}{l}\text { Discussion, } \\
\text { Pt has } \\
\text { final call }\end{array}$ & $\begin{array}{l}\mathbf{P t} \\
\text { asked } \\
\text { for } R \mathbf{x}\end{array}$ \\
\hline Dr decision, no discussion & $104(12)$ & $61(7)$ & I (0) & $\mathrm{I}(0)$ \\
\hline Discussion, Dr has final call & $24(3)$ & $597(7 \mathrm{I})$ & $31(4)$ & $0(0)$ \\
\hline Discussion, $\mathrm{Pt}$ has final call & $\mathrm{I}(0)$ & $12(1)$ & $9(1)$ & $0(0)$ \\
\hline Pt asked for $\mathrm{Rx}$ & $0(0)$ & $0(0)$ & $0(0)$ & $\mathrm{I}(0)$ \\
\hline
\end{tabular}

Notes: Values presented are $\mathrm{n}(\%)$, unless otherwise stated; percentages are calculated following the exclusion of missing data and are subject to rounding. Data were obtained from matched patient record forms and patient self-completion forms.

Abbreviations: Dr, doctor; PRF, patient record form; PSC, patient self-completion questionnaire; Pt, patient; Rx, prescription. 


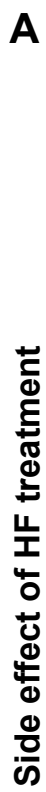

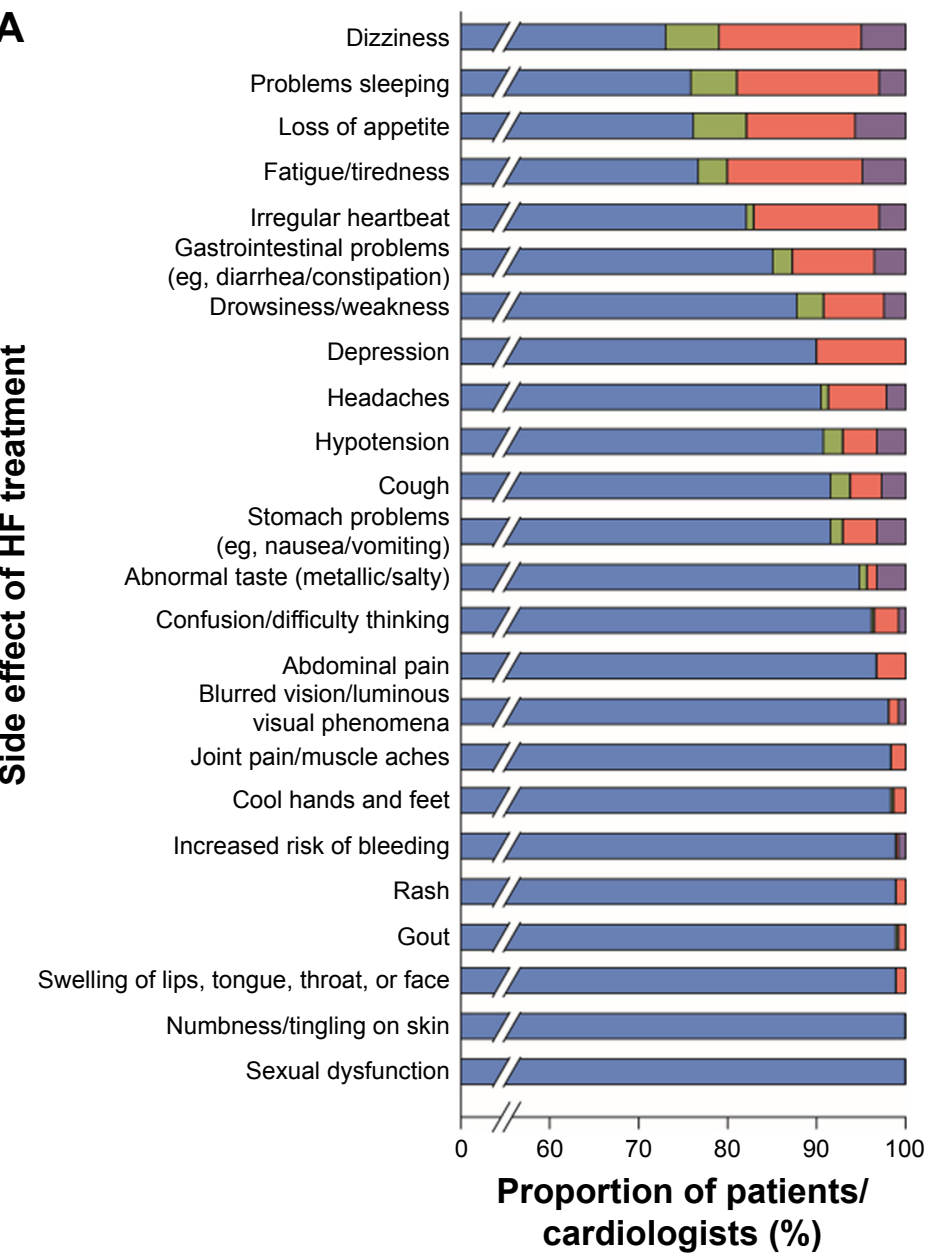

$\square$ Concordant - no side effect reported (a)

$\square$ Concordant - side effect reported (b)

$\square$ Discordant - side effect reported by patient, but not cardiologist (c)

$\square$ Discordant - side effect reported by cardiologist, but not patient (d)
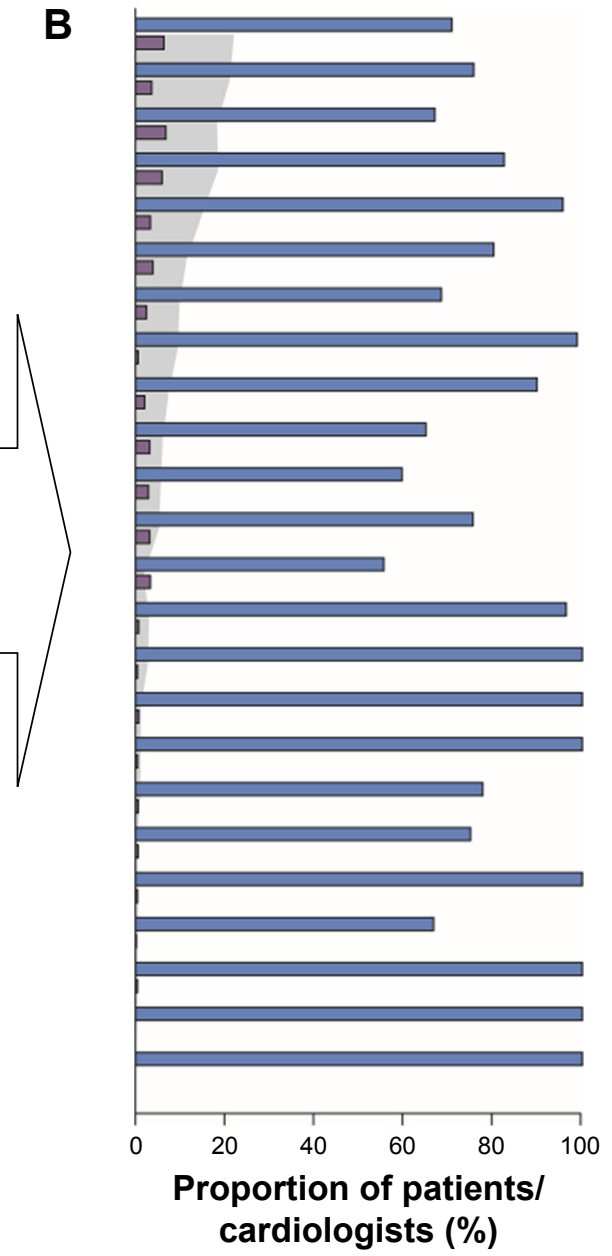

Patient-reported occurrence of side effects

Cardiologist underreporting of patients' side effects $\quad=c /(c+b)$

$\square$ Cardiologist overreporting of

patients' side effects $\quad=d /(d+a)$

Figure 4 Matched patient-cardiologist responses on the occurrence of side effects of HF treatments.

Notes: (A) Patient-cardiologist concordance of the occurrence of side effects of current HF treatment. (B) Cardiologist under- and overestimation of the occurrence of side effects of current HF treatment in relation to patient-reported occurrence.

Abbreviation: HF, heart failure.

or due to $\mathrm{HF}$ (median $=0$ visit), respectively (Figure 5 ). The corresponding proportions of patients experiencing $\geq 1$ allcause and HF-related outpatient and emergency room visit were $99 \%$ (median=11 visits) and $97 \%$ (median $=7$ ), and $31 \%$ (median $=0$ ) and $28 \%$ (median $=0$ ), respectively. Information on hospital readmission was available for 590 patients; of the patients hospitalized due to $\mathrm{HF}, 16 \%$ were readmitted to hospital within 30 days.

\section{Discussion}

This survey assessed care pathways of patients with HF in a real-world setting in China and provides evidence of HF treatment patterns in clinical practice. According to the population sampled in this study, cardiologists are the main physician speciality involved in first consultations of patients with a suspicion of HF, as well as in confirming HF diagnoses and initiating treatment; there is little involvement of family physicians, with only $10 \%$ being responsible for a first consultation and even fewer involved in diagnosis and treatment. These findings reflect the fact that involvement of primary care in HF management programs in China is extremely limited ${ }^{19}$ and differ from studies of HF care pathways in Western countries, ${ }^{7,8}$ where the family physician acts as a primary contact for patients with HF and will refer patients to specialists for further input when needed. Shared decision making is important for patient-centered care, whereby the physician educates the patient about treatment options, possible outcomes, and side effects, in order to 


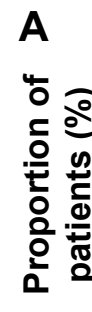

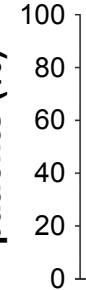

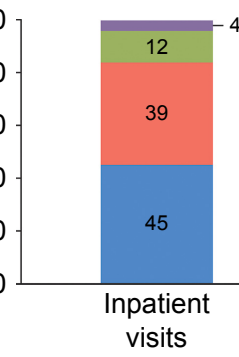

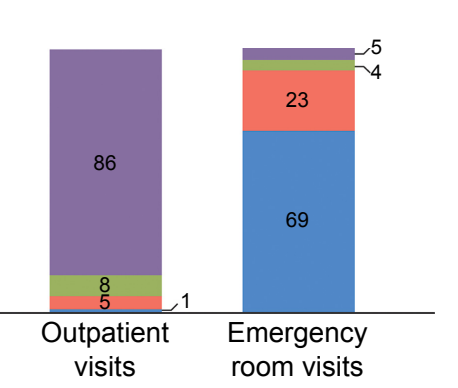

B

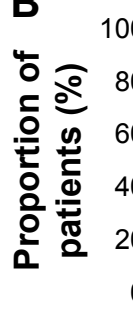

$\square$ event $\square 1$ events

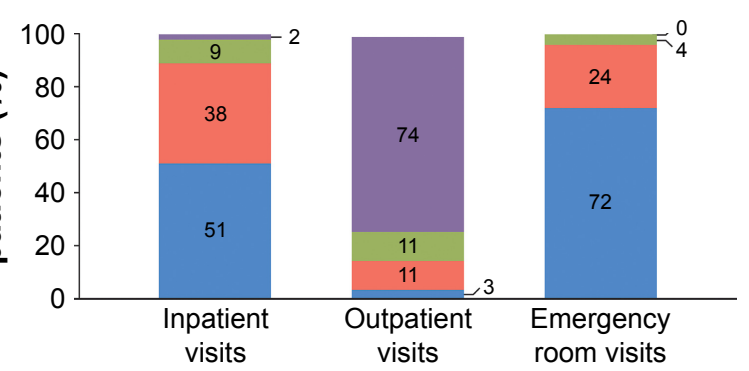

visits

\section{.}

Figure 5 Number of inpatient visits, outpatient visits, and emergency room visits per patient in the 12 months before the survey was conducted (A) for any reason and (B) due to HF.

Notes: $n=I, 286$ for all-cause inpatient visits; $n=I, 278$ for HF-related inpatient visits; $n=I, 490$ for all-cause outpatient visits; $n=I, 462$ for HF-related outpatient visits; $n=I, 467$ for all-cause emergency room visits; $n=I, 448$ for HF-related emergency room visits. Data were obtained from patient record forms.

Abbreviation: HF, heart failure.

reach an informed treatment decision. This study shows that even when a discussion occurs, the physician is most often the final decision maker for treatment decisions. Another important finding of this study relates to underreporting of adverse events by cardiologists. Improved communication between patients and cardiologists is essential to optimize treatment decision making and to increase awareness of the occurrence of treatment side effects.

The Chinese Society of Cardiology guidelines, similar to those published by the European Society of Cardiology, recommend that diagnosis of patients with HF should involve an echocardiogram, electrocardiogram, laboratory tests, and a chest X-ray. In the current study, the use of these diagnostic tests and tools was relatively high. Higher utilization rates of these diagnostic examinations by cardiologists in a hospital setting compared with family physicians in an office-based setting are not unexpected. ${ }^{20}$ Furthermore, the use of guideline-recommended HF drug classes, such as ACEis, ARBs, and BBs, was high and in line with the rates observed in specialist settings in Western countries. ${ }^{21-23}$ However, less than a third of patients with HFrEF received an MRA; this is notably lower than the rates reported for patients with HFrEF receiving care in specialist settings in Western countries ( $40 \%$ in the USA and $\sim 60 \%$ in European counties) ${ }^{21-23}$ highlighting an important gap exists in the utilization of a foundational disease-modifying therapy in HFrEF. In addition, less than half of patients with HFrEF were receiving the guidelinerecommended combination of $\mathrm{ACE} \mathrm{A} / \mathrm{ARB}+\mathrm{BB}$ and just one in five received a combination of $\mathrm{ACEi} / \mathrm{ARB}+\mathrm{BB}+\mathrm{MRA}$ (which is recommended for patients with HFrEF who remain symptomatic on ACEi/ARB+BB). ${ }^{24}$ These findings are in line with a recent study conducted in China by Huang et al which used national claims data and reported that $48 \%$ and
$23 \%$ of patients with HF were receiving these treatment combinations, respectively. ${ }^{25}$ The HF phenotype of patients included in the study by Huang et al was unspecified, so it might be expected that the proportion of patients with HFrEF who were receiving these combinations would be higher than for the overall study population. Nonetheless, the findings of the current study demonstrate that adherence to guidelines in China is suboptimal and should be improved.

The current study surveyed cardiologists, who may adhere more closely to clinical guidelines than family physicians. For example, in a review conducted by Jiang and Ge, it was reported that physicians in small hospitals and community hospitals may not follow guidelines regarding the use of BBs and ACEis. ${ }^{10}$ In addition, the cardiologists in the current study were practising within tier-2 and -3 hospitals; therefore, the findings may not be generalizable to care in rural settings or in smaller hospitals as assessed by Jiang and Ge. ${ }^{10}$ Indeed, previous studies have shown that there is great disparity in the use of HF treatments between developed and developing areas in China. ${ }^{14} \mathrm{~A}$ large survey conducted by Cao et al found that BBs and ACEis were used in 50\% and 90\% of patients in the Hunan province, respectively; however, in the Qinghai and Guizhou provinces, these drugs were used in $30 \%-33 \%$ and $33 \%-80 \%$ of patients, respectively. ${ }^{14}$ This imbalance in China with regard to access and utilization of health care between wealthier (usually urban) and poorer (usually rural) areas has long been recognized. ${ }^{26}$

The disease burden due to hospitalizations in the current study was overall high compared to those reported in Western studies. ${ }^{27,28}$ However, the total resource utilization of the patients from the study remains elusive, since in China, patients often receive care from multiple doctors and/or hospitals and doctors do not have access to patients' medical 
records of their visits to other hospitals. Therefore, the cardiologists in the current study might only have known the number of visits to the hospital in which they were treating the patient. In contrast, Huang et al analyzed data from a national claims database (covering all of a patient's hospitalizations regardless of at which hospital they took place) and reported higher hospitalization rates, as expected. ${ }^{25}$ In this study, $16 \%$ of patients who had been hospitalized for HF were readmitted within 30 days of discharge. This value is in line with the $15 \%$ reported by Huang et $\mathrm{al}^{25}$ and is within the range of 30-day readmission rates reported in the studies of Medicare patients with HF aged $\geq 65$ years in the USA $(11 \%-25 \%),{ }^{29-32}$ suggesting that patients in China with HF experience similar rates of hospital readmission to patients in Western countries.

The inevitable limitations associated with data collected from surveys are relevant for the current study, including recall bias, missing data, and overreporting of surveyed events. Nevertheless, the Adelphi DSP is an established method for investigating real-world treatment practices across a wide range of disease areas..$^{33}$

The patient population included in the study represents a sample of consulting patients with HF who are treated in a cardiology setting, and not those managed in primary care or by other specialities such as internal medicine or geriatricians. Only patients receiving care in tier-2 or -3 hospitals were included in the current study; these are more commonly found in large cities, and therefore, may not reflect patients receiving care in tier-1 hospitals, which are usually found in rural areas. Furthermore, in the current study, the split of tier- 2 and -3 hospitals was $31 \%$ and $69 \%$, respectively, which does not reflect the split in the Chinese health care system ( $78 \%$ vs $21 \%$ ), and may therefore impact the generalizability of the data. ${ }^{6}$

Although cardiologists were requested to collect PRF data on a series of consecutive patients to avoid selection bias, formal source data verification (ie, checks against patient medical records) was not performed. Moreover, diagnosis in the target patient group is based primarily on the judgment and diagnostic skills of the respondent cardiologist rather than on a diagnostic checklist, although patients are managed in accordance with the same routine diagnostic procedures representative of that clinical practice setting. More complex diagnostic procedures or methods for assessing functional status (eg, cardiopulmonary exercise testing) were not captured in our research; more research is needed to understand the impact of holistic integrative medicine on physician's choice of treatments. Choice of treatments given for HF may have been influenced by the presence of different comorbid conditions; yet, our questionnaire was unable to detect such potential interactions. In this respect, the cardiologists' responses are likely to reflect real-world data.

The Chinese guidelines recommend a 50\% ejection fraction cut-off to differentiate HFrEF from HFpEF phenotypes, which likely results in an overestimation of the HFrEF prevalence as compared to other geographies where $40 \%$ is used as the cut-off.

The selection of patients favors inclusion of those who consult more frequently, so patients with more severe disease or complications may be overrepresented in this sample. In addition, the inclusion criteria of this study required each cardiologist to recruit 10 patients, half of whom had HFrEF and the other half had HFpEF. Because there is an equal distribution of HF phenotype in the general HF population in China, ${ }^{3-5}$ this would be unlikely to introduce bias.

\section{Conclusion}

The results of this study allow for a better understanding of how patients with HF are diagnosed and treated, the extent to which patients influence treatment decisions, and how adverse events perceived by patients are recognized by the treating physicians in the sample of patients treated by cardiologists from China primarily practicing at tier- 2 and -3 hospitals in large cities. Although use of guideline-recommended diagnostic procedures was high, guideline-recommended double-combination therapy was prescribed to only half of the patients and triple combination to only one-fifth of the patients in the survey. A high burden of disease was observed with around half of the patients experiencing a hospitalization for $\mathrm{HF}$ in the 12 months before the survey. Finally, a high level of concordance was observed between matched cardiologist and patient pairs. One-fifth of the patients reported that they were not consulted regarding the choice of therapy, and that cardiologists tended to underestimate patientreported side effects. In conclusion, this study reinforces an unmet need in the care for patients with HF and suggests that improved communication is needed to optimize treatment decision making and to increase awareness of treatment side effects. Further studies with larger and more representative samples are needed to extrapolate these findings to the overall population of patients with HF in China.

\section{Acknowledgments}

This study was funded by Novartis Pharma AG, Basel, Switzerland. The manuscript was developed with important intellectual input from Heike Schwende and Daniel Viriato 
of Novartis Pharma AG, Basel, Switzerland. Medical writing support was provided by Carly L Sellick, BSc, of PharmaGenesis London, London, UK, and was funded by Novartis Pharma AG, Basel, Switzerland.

\section{Disclosure}

James DS Jackson and Sarah E Cotton are employees of Adelphi Real World, and were contracted by Novartis to conduct this study. Sara Bruce Wirta is an employee of Novartis Sweden AB; Raquel Lahoz, Bogdan Balas, and Frederico J Calado are employees of Novartis Pharma AG; and Milun Zhang is an employee of Novartis Pharma, China. Catia C Proenca is an employee of Wellmera AG contracted by Novartis Pharma AG. The authors report no other conflicts of interest in this work.

\section{References}

1. Global Burden of Disease Study 2013 Collaborators. Global, regional, and national incidence, prevalence, and years lived with disability for 301 acute and chronic diseases and injuries in 188 countries, 1990-2013: a systematic analysis for the Global Burden of Disease Study 2013. Lancet. 2015;386(9995):743-800.

2. Gu DF, Huang GY, Jiang HE, et al. Investigation of prevalence and distributing feature of chronic heart failure in Chinese adult population. Chin J Cardiol. 2003;31(1):3-6.

3. Ma JP, Wang L, Dang Q, et al. Retrospective analysis of drug treatment on inpatients with chronic heart failure. Chin J Epidemiol. 2007; 28(1):78-82.

4. Shanghai Investigation Group of Heart Failure. The evolving trends in the epidemiologic factors and treatment of hospitalized patients with congestive heart failure in Shanghai during the years of 1980,1990 and 2000. Chin J Cardiol. 2002;30:24-26.

5. Collaborative Group on Survey of Heart Failure in Shanghai. Crosssectional survey on the current status of drug therapy in patients with stable heart failure in Shanghai. Chin J Cardiol. 2001;29:644-648.

6. National Health and Family Planning Commission (NHFPC). The number of national medical and health institutions as of the end of June 2016. Available from: http://www.nhfpc.gov.cn/mohwsbwstjxxzx/s7 967/201608/87343c7d63ce41ca8d8fddf7a5db66b7.shtml. Accessed August 4, 2017.

7. Mehrhof F, Loffler M, Gelbrich G, et al; Competence Network Heart Failure. A network against failing hearts - introducing the German "Competence Network Heart Failure”. Int J Cardiol. 2010;145(1):135-138.

8. Tebbe U, Tschope C, Wirtz JH, et al. Registry in Germany focusing on level-specific and evidence-based decision finding in the treatment of heart failure: REFLECT-HF. Clin Res Cardiol. 2014;103(8): 665-673.

9. Chinese Society of Cardiology of the Chinese Medical Association, Editorial Board of the Chinese Journal of Cardiology. Chinese heart failure diagnosis and treatment guidelines; 2014. Available from: $\mathrm{http}: / /$ www.cjcv.org.cn/xinxueguan20144202/33844.htm?locale=zh_CN. Accessed August 4, 2017.

10. Jiang H, Ge J. Epidemiology and clinical management of cardiomyopathies and heart failure in China. Heart. 2009;95(21):1727-1731.

11. Society of Cardiology, Chinese Medical Association. Retrospective investigation of hospitalized patients with heart failure in some parts of China in 1980, 1990 and 2000. Chin J Cardiol. 2002;30:450-454.

12. Weihu F. [Cross-sectional survey on the current status of drug therapy in patients with stable heart failure in Shanghai]. Zhonghua Xinxueguanbing. 2001;29(11):644-648. Chinese.
13. Ma X, Xiang YT, Li SR, et al. Prevalence and sociodemographic correlates of depression in an elderly population living with family members in Beijing, China. Psychol Med. 2008;38(12):1723-1730.

14. Cao YM, Hu DY, Wang HY, Wu Y. [A survey of medical therapies for chronic heart failure in primary hospitals in China]. Zhonghua Nei Ke Za Zhi. 2006;45(11):907-909. Chinese.

15. Anderson P, Benford M, Harris N, Karavali M, Piercy J. Real-world physician and patient behaviour across countries: disease-specific programmes - a means to understand. Curr Med Res Opin. 2008; 24(11):3063-3072.

16. Jackson J, Cotton S, Bruce Wirta S, et al. Burden of heart failure on patients in China: results from a cross-sectional survey. Drug Des Devel Ther. 2018;12:1659-1668.

17. European Society for Opinion and Marketing Research (ESOMAR). International Code of Marketing and Social Research Practice 2007. Available from: http://www.icc.se/reklam/english/engresearch. Accessed August 22, 2013.

18. U.S. Department of Health \& Human Services. Summary of the HIPAA Privacy Rule, July 2013. Availble from: https://www.hhs.gov/ hipaa/for-professionals/privacy/laws-regulations/index.html. Accessed January 24, 2018.

19. Gu M, Ma Y, Zhou T, Xia Y. Evaluation of a community health service center-based intervention program for managing chronic heart failure. Balkan Med J. 2016;33(1):45-51.

20. Bohlken J, Kostev K. [Diagnostic and prescription behavior of general practitioners and specialist physicians in patients with dementia in 2005 and 2015 in Germany]. Psychiatr Prax. 2018;45(3):154-159. German.

21. Maggioni AP, Anker SD, Dahlstrom U, et al; Heart Failure Association of the ESC. Are hospitalized or ambulatory patients with heart failure treated in accordance with European Society of Cardiology guidelines? Evidence from 12,440 patients of the ESC Heart Failure Long-Term Registry. Eur J Heart Fail. 2013;15(10):1173-1184.

22. Crespo-Leiro MG, Segovia-Cubero J, Gonzalez-Costello J, et al; Project research team. Adherence to the ESC heart failure treatment guidelines in Spain: ESC Heart Failure Long-term Registry. Rev Esp Cardiol (Engl Ed). 2015;68(9):785-793.

23. von Scheidt W, Zugck C, Pauschinger M, et al. Characteristics, management modalities and outcome in chronic systolic heart failure patients treated in tertiary care centers: results from the EVIdence based TreAtment in Heart Failure (EVITA-HF) registry. Clin Res Cardiol. 2014; 103(12):1006-1014.

24. Ponikowski P, Voors AA, Anker SD, et al; ESC Scientific Document Group. 2016 ESC guidelines for the diagnosis and treatment of acute and chronic heart failure: the Task Force for the diagnosis and treatment of acute and chronic heart failure of the European Society of Cardiology (ESC) developed with the special contribution of the Heart Failure Association (HFA) of the ESC. Eur Heart J. 2016;37(27): 2129-2200.

25. Huang J, Yin H, Zhang M, Ni Q, Xuan J. Understanding the economic burden of heart failure in China: impact on disease management and resource utilization. J Med Econ. 2017;20(5):549-553.

26. Meng Q, Xu L, Zhang Y, et al. Trends in access to health services and financial protection in China between 2003 and 2011: a cross-sectional study. Lancet. 2012;379(9818):805-814.

27. Parrondo J, Sarria A. Heart failure in a health area of Madrid, Spain. Description and management from electronic medical records. Value Health. 2015;18(7):A381-A382.

28. Aydogdu AD, Bozkurt E, Cavusoglu Y, et al. The direct cost components in heart failure with reduced ejection fraction (HF-REF) in Turkey: the results of a Delphi panel. Abstract presented at: ISPOR 19th Annual European Congress; November, 2016; Vienna, Austria.

29. Dharmarajan K, Hsieh AF, Lin Z, et al. Diagnoses and timing of 30-day readmissions after hospitalization for heart failure, acute myocardial infarction, or pneumonia. JAMA. 2013;309(4):355-363. 
30. Chen J, Ross JS, Carlson MD, et al. Skilled nursing facility referral and hospital readmission rates after heart failure or myocardial infarction. Am J Med. 2012;125(1):100.e1-e9.

31. Krumholz HM, Merrill AR, Schone EM, et al. Patterns of hospital performance in acute myocardial infarction and heart failure 30-day mortality and readmission. Circ Cardiovasc Qual Outcomes. 2009; 2(5):407-413.
32. Ross JS, Chen J, Lin Z, et al. Recent national trends in readmission rates after heart failure hospitalization. Circ Heart Fail. 2010;3(1):97-103.

33. Anderson P, Benford M, Harris N, Karavali M, Piercy J. Real-world physician and patient behaviour across countries: Disease-Specific Programmes - a means to understand. Curr Med Res Opin. 2008;24(11): 3063-3072.

\section{Publish your work in this journal}

Drug Design, Development and Therapy is an international, peerreviewed open-access journal that spans the spectrum of drug design and development through to clinical applications. Clinical outcomes, patient safety, and programs for the development and effective, safe, and sustained use of medicines are the features of the journal, which has also been accepted for indexing on PubMed Central. The manuscript management system is completely online and includes a very quick and fair peer-review system, which is all easy to use. Visit http://www.dovepress.com/testimonials.php to read real quotes from published authors.

Submit your manuscript here: http://www.dovepress.com/drug-design-development-and-therapy-journal 